Various techniques have" been tried to allow easy lengthening of the electrode wire. These include:

1. Leaving a loop of wire in the heart (in two out of three the electrode tip displaced);

2. Manually feeding more wire into the vein from spare loops retained in a subcutaneous nylon sleeve;

3. Initial suturing of the electrode tip to the endocardium, allowing spontaneous feeding of excess wire from a nylon bag into the vein, as the electrode straightens with growth.

Endocardial pacing seems a feasible long-term approach in children.

\section{A DECADE OF THORACIC PAPERS}

A. JOHN ROBERTSON During the last 10 years, over 700 papers have been received by the medical editor of Thorax. About $53 \%$ of these have been from the United Kingdom and $45 \%$ from 40 different overseas countries. About $40 \%$ of all papers were rejected, the acceptance rate being $64 \%$ from Great Britain and $56 \%$ from elsewhere. The countries with the highest acceptance rate were New Zealand and Australia: some examples of percentages from other places are shown, as well as some reasons for rejection. In 1960 the journal contained 360 pages, but in 1970, 768 pages are scheduled. The change in subject matter is briefly discussed, as well as methods of assessing and editing papers.

\section{BUSULPHAN LUNG}

\section{Clinical Features}

W. A. LITTLER Diffuse pulmonary fibrosis is now a recognized complication of busulphan therapy. Heard and Cooke (1968) examined the lungs of 14 patients dying of chronic myeloid leukaemia and found histological changes attributable to busulphan in six, but in only one of these had busulphan lung been suspected during life. This high incidence of busulphan lung at necropsy has prompted a prospective study to discover whether the condition can be detected earlier by means of serial clinical, radiographic and physiological examinations. The methods employed in this study and the preliminary findings are reported along with details of the first patient from the series in whom busulphan lung was diagnosed during life and confirmed at necropsy. This patient was a man of 61 years with chronic myeloid leukaemia treated over a period of 19 months with a total dose of $1,000 \mathrm{mg}$. busulphan. Evidence of pulmonary disease did not arise until one month after withdrawal of busulphan, and consisted of intense dyspnoea, a dry cough, central cyanosis and persistent crepitations at the lung bases. The chest radiograph showed a progressive diffuse mottling throughout both lungs and physiological tests demonstrated a restrictive ventilatory defect associated with a gas transfer factor of only $25 \%$ of the predicted normal. The pulmonary condition progressed to a fatal termination within three months of its onset despite the fact that busulphan had been withheld and large doses of prednisone given.

\section{Histopathology}

P. S. HASLETON Patients treated with busulphan may develop characteristic cellular and fibrous changes in their lungs. The alveoli are lined by large cells with big nuclei and copious cytoplasm which contains clear bodies resembling vacuoles. The cells desquamate into the alveolar spaces and disintegrate to form an amorphous eosinophilic debris. The identification of these cells and the ultrastructure of the debris are described in the following paper. The alveolar walls become thickened by chronic oedema and then interstitial fibrosis. Organization of the intra-alveolar debris also occurs. In the case described pulmonary cholesterol-ester granulomas were also present. They consisted of fibrous nodules around acicular cholesterol clefts with a surrounding chronic inflammatory exudate. The hypothesis is advanced that such granulomas are associated more with hyperplasia of granular pneumocytes than with pulmonary hypertension.

\section{Electron Microscopy}

J. M. KAY Electron microscopy was carried out to identify the large alveolar cells and to determine the nature of the intra-alveolar debris which characterize the histological picture of this condition. The enlarged alveolar cells had the ultrastructural features of granular (type II) pneumocytes. They possessed prominent intracytoplasmic lamellar secretory inclusions (lamellar bodies), and their free borders showed numerous irregular, short microvilli. The intra-alveolar debris consisted of strands of amorphous electron-dense fibrillary material interspersed with roughly circular bodies composed of concentric electron-dense membranes. The fibrillary material resembled fibrin and the circular bodies appeared identical to the lamellar secretory inclusion bodies of granular pneumocytes. In some instances, it appeared that granular pneumocytes had disintegrated and liberated their lamellar bodies, which had given rise to the intra-alveolar debris. Also scattered within the alveolar spaces were myelin figures which resembled phospholipid membranes. Evidence of organization of the intra-alveolar debris was seen in the form of groups of fibrils showing the characteristic periodic transverse striation of collagen.

\section{PROSTHETIC TRACHEAL REPLACEMENT}

JOHN BORRIE The principles governing successful tracheal reconstruction are reviewed. Over a 15-year experimental experience of reconstructing long tracheal defects $(5 \mathrm{~cm}$. or more), the methods of suturing alone, suturing with lateral relieving incisions, the use of ribbed Dacron prostheses, Marlex mesh, and one form of Silastic prosthesis have all been tried and rejected. More promising results from current research methods are shown. 